\title{
A Rare Case of Abacavir Hypersensitivity Syndrome
}

\author{
Charles-Lwanga Bennin, MD
}

\section{Case}

A 56-year-old African American male with a history of type II diabetes mellitus complicated by neuropathy, infections with human immunodeficiency virus (HIV), with a known CD4 count of 25 per cubic millimeter, hepatitis $B$ and hepatitis $C$ presented with six months of generalized weakness over his lower extremities, poor balance, nausea and vomiting. The patient had recently started anti-retroviral therapy (ART) and noticed that the onset of his symptoms coincided with the initiation of this therapy. He was taking Trizivir, which consists of abacavir (300mg each day), lamivudine (150mg each day), and zidovudine (300mg each day), atazanavir (300mg each day) and ritonavir (100mg each day). The patient was also taking trimethoprim/sulfamethoxazole (TMP-STX) and fluconazole as prophylaxis against opportunistic infections. He described his generalized weakness as musculoskeletal and involving all his extremities, especially his legs: it was exacerbated with activity and relieved with rest. This weakness had led to gait instability without falls. He noted that his nausea was constant and had resulted in non-bilious, non-bloody emesis. He vomited approximately 2-3 times per day for 3 days. As a result, he had poor oral intake. He denied any fevers but he did report chills. He also reported a 40-lb weight loss over 3 months. Of note, he noticed that his symptoms diminished when he stopped taking of his ART medications. As a result, the patient had been non-compliant with his ART. He denied any significant alcohol consumption or recreational drugs use.

On examination, the patient was febrile to $100{ }^{4}$ degrees Fahrenheit, tachycardic to 104 beats per minute, and mildly hypertensive to $138 / 79$. He had no lymphadenopathy; his cardiovascular exam was tachycardic but otherwise within normal limits; and his pulmonary exam revealed basilar crackles. His abdominal exam was unremarkable. The patient's neurologic exam revealed no cranial nerve deficits or ataxic gait. Strength was preserved in all extremities with no sensory deficits. On admission, his laboratory work up (Table 1) was significant for normocytic anemia, leukocytosis, hyperkalemia, hyponatremia and elevated creatinine.

\section{Hospital Course}

The patient was diagnosed with probable abacavir hypersensitivity. This was a clinical diagnosis in the setting of a negative infectious workup. His ART was discontinued. The patient was tested for the HLA-B ${ }^{\star} 5701$ allele. This allele, which has an association with abacavir hypersensitivity, was negative in the patient. Over the following thirty six hours his complaints gradually improved. He regained his strength and had complete resolution of his nausea and vomiting before discharge.

\section{Discussion}

Abacavir is a nucleoside reverse transcriptase inhibitor used as part of the ART regimen in treating patients with HIV. It has been in use since 1998. Abacavir is an important guanosine analog that targets reverse transcriptase with high bioavailability and the ability to cross the blood brain barrier. Its use as a once daily medication helps encourage compliance among patients.

Abacavir hypersensitivity syndrome (HRS) is a sub-type of drug hypersensitivity syndrome. The syndrome is an idiosyncratic reaction with a delayed onset that can be fatal ${ }^{1}$. The symptoms associated with abacavir hypersensitivity syndrome result from an immunologically mediated hypersensitivity reaction involving the major histocompatibility complex class I allele HLA-B*5701. An overriding theory involves abacavir stimulating antigen specific HLA-B ${ }^{\star} 5701$ restricted CD8+ T Cells ${ }^{2}$. For other drugs, hypersensitivity syndrome may occur as the result of genetic deficiency of a detoxifying enzyme,

\section{Table 1. Admission Laboratory Values and Reference Range in Adults (Righthand column)}

\begin{tabular}{|c|c|c|c|c|c|}
\hline WBC & $12.9 \mathrm{~K} / \mathrm{mm}^{3}$ & $3.6-10.6$ & Glucose & 98 mg/dL & $74-118$ \\
\hline RBC & $2.75 \mathrm{M} / \mathrm{mm}^{3}$ & $4.70-5.43$ & Bun & $37 \mathrm{mg} / \mathrm{dL}$ & $8-20$ \\
\hline HGB & $8.4 \mathrm{gm} / \mathrm{dl}$ & $12.3-16.3$ & Creatinine & $1.73 \mathrm{mg} / \mathrm{dL}$ & $0.7-1.2$ \\
\hline HCT & $23.8 \%$ & $35.5-52$ & Sodium & $128 \mathrm{mmol} / \mathrm{L}$ & $136-144$ \\
\hline MCV & 86.6 FL & $82.7-97.7$ & Potassium & $5.7 \mathrm{mmol} / \mathrm{L}$ & $3.6-5.1$ \\
\hline RDW & $15.0 \%$ & $11.5-16.5$ & Chloride & $94 \mathrm{mmol} / \mathrm{L}$ & $101-111$ \\
\hline PLT & $267 \mathrm{~K} / \mathrm{mm}^{3}$ & $150-430$ & Carbon Dioxide & $20 \mathrm{mmol} / \mathrm{L}$ & $22-32$ \\
\hline MPV & $8.6 \mathrm{fl}$ & $6.2-10.0$ & Calcium & $8.5 \mathrm{mg} / \mathrm{dL}$ & $8.9-10.3$ \\
\hline CD4+ & 25 Cells/uL & $594-1663$ & & & \\
\hline CD8+ & 301 Cells/uL & $272-932$ & & & \\
\hline
\end{tabular}


Table 2. Abacavir Hypersensitivity Treatment Recommendations, adapted from the Federal Drug Administration guidelines

Screening for the HLA-B ${ }^{\star} 5701$ allele is recommended for all patients prior to starting abacavir therapy.

Screening is recommended prior to reinitiation of abacavir in patients of unknown HLA-B` 5701 status who have previously tolerated abacavir.

For HLA-B*5701-positive patients, treatment with an abacavir-containing regimen is not recommended and should be considered only under exceptional circumstances when the potential benefit outweighs the risk.

HLA-B ${ }^{\star} 5701$-negative patients may develop a hypersensitivity reaction to abacavir; however, this occurs significantly less frequently than in HLA-B*5701-positive patients.

Discontinue abacavir therapy permanently if the patient becomes seriously ill and hypersensitivity cannot be ruled out, regardless of HLA-B*5701 status.

Following a hypersensitivity reaction to abacavir, NEVER restart abacavir or any abacavir-containing product because severe symptoms can occur within hours and may include life-threatening hypotension and death.

drug immunosuppression, drug-specific T cell or viral drug interactions ${ }^{1}$. This hypersensitivity reaction can occur up to six weeks after initiation of therapy.

Abacavir hypersensitivity syndrome is characterized by gastrointestinal symptoms including nausea, vomiting, abdominal cramping, respiratory symptoms including coughing and shortness of breath and constitutional symptoms including fever, body rash and weight loss ${ }^{3}$. Some studies have also linked abacavir to an increased risk of myocardial infarction ${ }^{4}$.

Abacavir HRS is a clinical diagnosis but there are tests that support the diagnosis. Along with an assay for the HLA-B ${ }^{\star} 5701$ allele, epicutaneous patch testing may be utilized ${ }^{1,2}$. The method involves the application of antigen to the skin at standardized concentrations to the back or limbs. The ideal timing of the patch test readings is on day 2 and day 4, with additional reading at day 6 or 7 which will pick up approximately $10 \%$ more positives that were negative at days 2 and 4 . This patch will produce both a visible response along with palpable skin changes. Studies have shown that all patients with abacavir HRS and a positive skin test all carry the HLA-B ${ }^{\star} 5701$ allele, however not all HLA-B ${ }^{\star} 5701$ allele carrying patients will have abacavir HRS. It is also important to note that while a positive epicutaneous patch testing confirms abacavir HRS, a negative result cannot rule out HRS. Abacavir HRS is often over diagnosed in African Americans; a low frequency of African Americans carry the HLA-B ${ }^{\star} 5701$ allele $^{5}$.
Treatment of abacavir HRS involves stopping administration of abacavir. It is important not to re-challenge a patient with a positive clinical reaction as there is the possibility of increased morbidity and death. Avoiding abacavir in patients with HLA-B ${ }^{\star} 5701$ has been shown to reduce the incidence of this hypersensitivity syndrome. Although ART regimen initiation should be left to infectious disease specialists, it is important to be aware of the recommendations by the FDA pertaining to abacavir HRS (Summarized in Table 2) ${ }^{2}$.

\section{References}

1. Santiago, F., Gonçalo, M., Vieira, R., et al. Epicutaneous patch testing in drug hypersensitivity syndrome (DRESS). Contact Dermatitis 2010: 62:1:47-53

2. Mallal, S., Phillips, E., Carosi, G., HLA-B ${ }^{\star} 5701$ Screening for Hypersensitivity to Abacavir. N Engl J Med 2008;358:568-79

3. Hughes, C., Foisy, M., Dewhurst, N.,et al. Abacavir Hypersensitivity Reaction: an Update. The Annals of Pharmacotherapy, 2008: 44:387-396.

4. D:A:D Study Group. Use of nucleoside reverse transcriptase inhibitors and risk of myocardial infarction in HIV-infected patients enrolled in the D:A:D study. The Lancet, 2008:371:9622:1417 - 1426.

5. Saag, M., Balu, R., Phillips, E, et al. High Sensitivity of Human Leukocyte Antigen- $\mathrm{B}^{\star} 5701$ as a Marker for Immunologically Confirmed Abacavir Hypersensitivity in White and Black Patients. Clinical Infectious Diseases (CID), 2008: 46:1111-1118.

6. www.fda.gov/Drugs/DrugSafety/PostmarketDrugSafetyInformationforPatientsandProviders/ucm123927. htm 